\title{
Intraductal tubulopapillary neoplasm accompanied by invasive carcinoma of the pancreas: A case report and review of the literature
}

\author{
LI NIU, ZHIGAO XU, HUAN LIU, HONG CAO and GUIFANG YANG \\ Department of Pathology, Zhongnan Hospital of Wuhan University, Wuhan, Hubei 430071, P.R. China
}

Received November 2, 2016; Accepted January 23, 2017

DOI: $10.3892 / \mathrm{mco} .2017 .1216$

\begin{abstract}
Intraductal tubulopapillary neoplasms (ITPNs) are rare pancreatic neoplasms accounting for $\sim 0.4 \%$ of pancreatic tumors. However, their clinicopathological characteristics have not been clearly determined and the number of available clinical studies on this type of tumor is limited at present. Due to the rare incidence of ITPN, diagnosis is often delayed. We herein present a unique case of a 38-year-old man who was diagnosed with ITPN accompanied with invasive carcinoma of the pancreas and underwent total pancreatectomy. The morphological characteristics of ITPN include closely packed tubular glands, without mucin secretion, accompanied with invasion of the loose connective tissue. The immunohistochemical staining suggested that the tumors did not originate from the gastrointestinal tract but rather from the bile duct. In addition, the Ki-67 positive staining rate of tumor cells was $<20 \%$. The microsatellite instability analysis demonstrated microsatellite stability, without detected gene mutations of epidermal growth factor receptor, Kirsten rat sarcoma viral oncogene homolog, neuroblastoma RAS viral oncogene homolog or B-Raf proto-oncogene. However, a mutation was identified in exon 9 of the P53 gene, the most frequently mutated gene in human cancer, which suggested the underlying mechanism of ITPN. On the basis of this case, the aim of this study was to summarize and review the relevant reports of ITPNs in recent years, in order to investigate the clinicopathological characteristics and differential diagnosis of ITPN.
\end{abstract}

\section{Introduction}

According to the World Health Organization (WHO), intraductal tubulopapillary neoplasm (ITPN) is a type of intraductal

Correspondence to: Dr Guifang Yang, Department of Pathology, Zhongnan Hospital of Wuhan University, 169 East Lake Road, Wuhan, Hubei 430071, P.R. China

E-mail: guifangyang61@163.com

Key words: intraductal tubulopapillary neoplasm, intraductal papillary mucinous neoplasm, microsatellite instability, P53, gene mutation epithelial tumor of the pancreas $(1,2)$. ITPNs are located in pancreatic ducts and arise from the pancreatic ductal epithelium. However, the lesions that originate in pancreatic ducts may be quite complex. Based on the intraductal papillary mucinous neoplasm (IPMN), the new term or classification for this intraductal epithelial tumor of the pancreas was ITPN. ITPNs displaying highly heteromorphic hyperplasia may be distinguished from IPMNs by gross morphology, clinicopathological characteristics and immunophenotype. Due to the rarity and lower incidence of ITPNs, which only represent $0.4 \%$ of all pancreatic tumors, the available data on the clinicopathological and molecular characteristics of this type of tumor are limited (1). The aim of the present study was to analyze the clinicopathological, immunophenotypic and molecular genetic characteristics of ITPNs to gain a better insight into this disease entity.

\section{Case report}

In October, 2015, a 38-year-old man presented to the Zhongnan Hospital (Wuhan, China) without any prior medical history of tiredness, anorexia, flatulence or abnormal findings on urinalysis. The physical examination was unremarkable, with the exception of mild jaundice of the sclerae. The results of the laboratory analysis were as follows: Total bilirubin, $125.9 \mu \mathrm{mol} / 1$ (normal range, 3.4-17.1 $\mu \mathrm{mol} / \mathrm{l}$ ); conjugated bilirubin, $77.8 \mu \mathrm{mol} / 1$ (normal range, 0-6 $\mu \mathrm{mol} / 1$ ); unconjugated bilirubin (normal range, 1.7-10.2 $\mu \mathrm{mol} / 1$ ), $48.1 \mu \mathrm{mol} / 1$; alanine aminotransferase, $261 \mathrm{U} / 1$ (normal range, 0-40 U/1); aspartate aminotransferase, $93 \mathrm{U} / 1$ (normal range, 0-45 U/1); $\gamma$-glutamyl transpetidase, 1,486 U/1 (normal range, 0-50 U/1); alkaline phosphatase, $180 \mathrm{U} / 1$ (normal range, 40-160 U/1); and total bile acid, $299.6 \mu \mathrm{mol} / 1$ (normal range, $0-10 \mu \mathrm{mol} / 1$ ). These abnormal biochemical indicators suggested obstructive jaundice. Computed tomography (CT) revealed a low-attenuation mass, sized $42 \times 40 \mathrm{~mm}$, located in the head of the pancreas. Patchy and heterogeneous shadowing of the portal artery and vein was accompanied by partial capsule-like rim enhancement of the surrounding tissue (Fig. 1A). There was no evidence of lymphadenectasis or metastasis to other organs, including the liver and lung. The intrahepatic and extrahepatic bile ducts and the main pancreatic duct were clearly dilated, with obvious atrophy of the pancreatic parenchyma (Fig. 1A). Consequently, classical pancreatoduodenectomy was performed, accompanied 

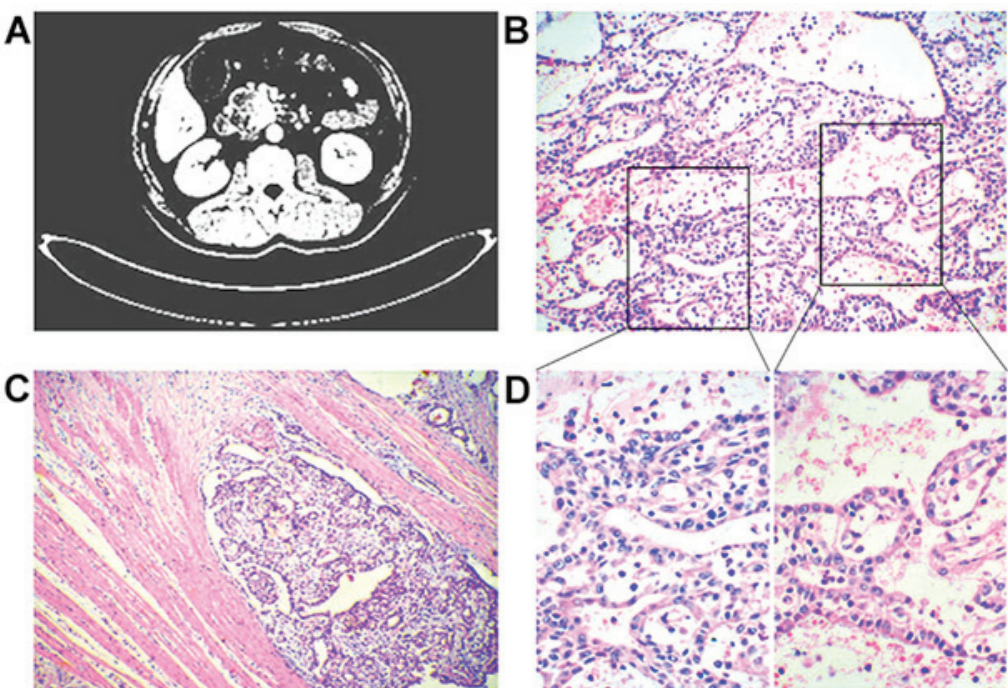

Figure 1. A 38-year-old patient with intraductal tubulopapillary neoplasm (ITPN). (A) The computed tomography scan in a 38 -year-old patient with intraductal tubulopapillary neoplasm (ITPN) revealed a round and inhomogeneous mass in the head of the pancreas measuring $4 \mathrm{~cm}$ in diameter. (B-D) Histological characteristics of ITPN: (B) Magnification, x100; (C) tissue invasion by the atypic neoplastic cells (magnification, x40); (D) higher magnification (x200) of the segments outlined in B.

with cholecystectomy. Macroscopically, a solid tumor sized $42 \times 40 \times 20 \mathrm{~mm}$ was identified in the head of the pancreas, with unclear borders. On cross-section the tumor was solid, gray and brown, and accompanied by cavitation, hemorrhage and necrosis. Mucin production was not observed in the tumor specimens.

The tumor specimens were fixed in a $4 \%$ solution of neutral formaldehyde. Following dehydration, the fixed specimens were embedded in paraffin and cut into 3-4- $\mu \mathrm{m}$ sections. The technique of EnVision Immunity immunohistochemical staining (ZSGB-BIO Co., Beijing, China) was applied with automated instrumentation. The antibodies in the EnVision staining procedure included antibodies targeted against cytokeratin (CK) (ZM-0069; mouse; dilution, 1:100), mucin (MUC) 1 and MUC2 (ZM-0391 and ZM-0392, respectively; mouse; dilution, 1:100), carcinoembryonic antigen (ZM-0062; mouse; dilution, 1:100), CD56 (ZM-0057; mouse; dilution, 1:100), chromogranin A (CgA) (ZM-0076; mouse; dilution, 1:100) and Ki-67 (ZM-0166; mouse; dilution, 1:100). All the antibodies were purchased from ZSGB-BIO. Detailed operating procedures were conducted according to the manufacturer's instructions. The resultant black and brown granules in the cells were considered as a positive reaction.

Microsatellite instability (MSI) analysis and fluorogenic quantitative polymerase chain reaction methods were applied to detect the expression of epidermal growth factor receptor (EGFR), Kirsten rat sarcoma viral oncogene homolog (KRAS), neuroblastoma RAS viral oncogene homolog (NRAS), B-Raf proto-oncogene (BRAF) and P53 gene mutations. Detailed operative procedures were conducted according to the manufacturer's instructions and standard operating procedures.

\section{Discussion}

An electronic search was performed through PubMed (National Library of Medicine, NIH, Bethesda, MD, USA) and the Chinese Periodical Database (search date up to and including June 6, 2016) for ITPN cases. In total, 44 English and 3 Chinese articles were identified using the key words 'intraductal tubulopapillary neoplasms'. After screening, which involved the deletion of significantly irrelevant information, 36 ITPN cases and 50 random cases of IPMN were selected.

Microscopically, the morphological characteristics of the tumor was basophilic cuboidal cells of uniform size in the pancreatic ducts, mostly arranged in a tubular and papillary pattern. The tumor cells were cuboidal, with intense nuclear staining and obvious presence of a nucleolus, exhibiting moderate dysplasia. The majority of the tumor cells were arranged in a cribriform or back-to-back pattern; additionally, normal pancreatic intraductal epithelium was clearly seen. The tubular glands were also closely packed, without secretion of mucin (Fig. 1B and D). The neoplastic cells had partially invaded the smooth muscle wall of the duodenum and were found proliferating around the small blood vessels and extending into the connective tissue (Fig. 1C).

Furthermore, immunohistochemical staining of the tumors showed positive reactions for CK and MUC1 and a partially positive reaction for vimentin, but not MUC2. The Ki-67 index was $20 \%$, indicating proliferative activity (Fig. 2). However, staining for CD56 and CgA was negative in the tumor specimens (Fig. 3).

MSI analysis revealed microsatellite stability (Fig. 4A); however, no gene mutations were detected for EGFR, KRAS, NRAS, BRAF and phosphoinositide-3-kinase, catalytic subunit $\alpha$ (Fig. 4B). In an alternate mechanistic pathway of tumor generation and associated mechanisms, immunohistochemical staining showed positive protein expression staining patterns for P53 of 50\% (data not shown) and P53 gene mutation analysis revealed a base mutation, namely a substitution of A with $\mathrm{T}$ in the 2,030 position of the exon 9 DNA sequence of the P53 gene in this tumor (Fig. 4C).

A comprehensive analysis that included the present case and other ITPN cases that were previously reported in the literature, suggested that there was immunoreactivity for $\mathrm{CK}$ 
Table I. Immunohistochemical and mutation analysis.

Immunohistochemical staining

Mutational analysis

Case $\begin{array}{lllllllllllllllllll}\text { MUC1 } & \text { MUC2 } & \text { MUC5AC } & \text { CK7 } & \text { CK19 } & \text { CDX2 } & \text { CgA } & \text { Ki-67(\%) } & \text { P53 } & \text { TP53 } & \text { KRAS NRAS } & \text { BRAF } & \text { PI3KCA }\end{array}$

\begin{tabular}{|c|c|c|c|c|c|c|c|c|c|c|c|c|c|c|}
\hline 1 & + & - & ND & - & ND & - & - & 20 & + & + & - & - & - & - \\
\hline 2 & + & - & - & ND & - & ND & ND & 30 & + & ND & + & ND & ND & ND \\
\hline 3 & + & - & - & ND & - & ND & ND & 35 & - & ND & + & ND & ND & ND \\
\hline 4 & + & - & - & ND & - & ND & ND & 40 & + & ND & - & ND & ND & ND \\
\hline 5 & + & - & - & ND & ND & ND & ND & 30.5 & - & ND & - & ND & ND & ND \\
\hline 6 & + & - & - & ND & ND & ND & ND & 6.1 & - & ND & - & ND & ND & ND \\
\hline 7 & + & - & - & ND & ND & ND & ND & 9.2 & - & ND & - & ND & ND & ND \\
\hline 8 & + & - & - & ND & ND & ND & ND & 21.4 & + & ND & - & ND & ND & ND \\
\hline 9 & + & - & - & ND & ND & ND & ND & 24.6 & - & ND & - & ND & ND & ND \\
\hline 10 & + & - & - & ND & ND & ND & ND & 19.1 & - & ND & - & ND & ND & ND \\
\hline 11 & + & - & - & ND & ND & ND & ND & 33.4 & - & ND & - & ND & ND & ND \\
\hline 12 & + & - & - & ND & ND & ND & ND & 43 & - & ND & - & ND & ND & ND \\
\hline 13 & + & - & - & ND & ND & ND & ND & 28.7 & - & ND & - & ND & ND & ND \\
\hline 14 & + & - & - & ND & ND & ND & ND & 10.8 & - & & - & ND & ND & ND \\
\hline 15 & + & - & - & + & + & ND & ND & 30.5 & ND & - & - & ND & - & - \\
\hline 16 & + & - & - & + & + & ND & ND & 6.1 & ND & - & - & ND & - & - \\
\hline 17 & + & - & - & + & + & ND & ND & 9.2 & ND & - & - & ND & - & - \\
\hline 18 & + & - & - & + & + & ND & ND & 21.4 & ND & + & - & ND & - & - \\
\hline 19 & + & - & - & + & + & ND & ND & 24.6 & ND & - & - & ND & - & + \\
\hline 20 & + & - & - & + & - & ND & ND & 19.1 & ND & - & - & ND & - & - \\
\hline 21 & + & - & - & + & - & ND & ND & 33.4 & ND & - & - & ND & - & + \\
\hline 22 & + & - & - & + & - & ND & ND & 43 & ND & - & - & ND & - & - \\
\hline 23 & + & - & - & + & - & ND & ND & 28.7 & ND & ND & ND & ND & - & - \\
\hline 24 & + & - & - & + & - & ND & ND & 10.8 & ND & - & ND & ND & ND & ND \\
\hline 25 & + & - & - & + & - & ND & ND & $5-20$ & ND & ND & ND & ND & ND & ND \\
\hline 26 & + & - & - & + & ND & ND & ND & $10-15$ & ND & - & - & ND & - & - \\
\hline 27 & + & ND & - & + & - & ND & ND & $20-30$ & ND & ND & - & ND & ND & ND \\
\hline 28 & + & - & - & ND & ND & ND & ND & $\mathrm{ND}$ & ND & ND & ND & ND & ND & ND \\
\hline 29 & + & - & - & + & + & ND & ND & 9 & ND & ND & - & ND & + & ND \\
\hline 30 & + & + & + & + & + & ND & ND & 32 & ND & + & - & ND & + & - \\
\hline 31 & + & - & - & + & + & ND & ND & 24.6 & ND & ND & ND & ND & ND & ND \\
\hline 32 & + & - & - & + & + & ND & ND & 5 & ND & - & - & ND & ND & ND \\
\hline 33 & + & - & - & + & + & ND & ND & ND & ND & - & ND & ND & ND & ND \\
\hline 34 & ND & - & - & + & + & ND & ND & ND & ND & ND & ND & ND & ND & ND \\
\hline 35 & + & - & - & + & + & ND & ND & ND & ND & ND & ND & ND & - & ND \\
\hline 36 & ND & ND & ND & + & + & ND & ND & $10-60$ & ND & + & - & ND & - & ND \\
\hline
\end{tabular}

MUC, mucin; CK, cytokeratin; CgA, chromogranin A; KRAS, Kirsten rat sarcoma viral oncogene homolog; NRAS, neuroblastoma RAS viral oncogene homolog; BRAF, B-Raf proto-oncogene; PI3KCA, phosphoinositide-3-kinase, catalytic subunit $\alpha$; ND, not determined.

and MUC1 in tumor specimens. A number of cases clearly expressed P53; by contrast, MUC2 and MUC5AC were not expressed (Table I). In addition, some cases exhibited mutations in both the KRAS and P53 genes (Table I).

A large body of the literature has previously demonstrated that tumor tissues in IPMN were mainly located in the duct, exhibiting a papillary arrangement. The branches of the papillary formation are intricate, and serve a function akin to sieves. Most cells in IPMN are mucous epithelial cells, including goblet cells, whereas only few cells are eosinophilic epithelial or cuboidal epithelial (pancreatic duct type epithelium). Among 50 randomly selected patients with IPMN (defined according to the WHO classification of digestive system tumors in 2010) 21 patients had low-grade intraepithelial neoplasia, 9 had high-grade intraepithelial neoplasia and 20 had invasive cancer. The clinicopathological characteristics differentiating IPMN and ITPN are summarized in Table II.

ITPN accounts for $\sim 0.4 \%$ of pancreatic tumors, and it is a rare pancreatic neoplasm with few reported cases investigating its clinical and molecular pathology (3). Due to the 

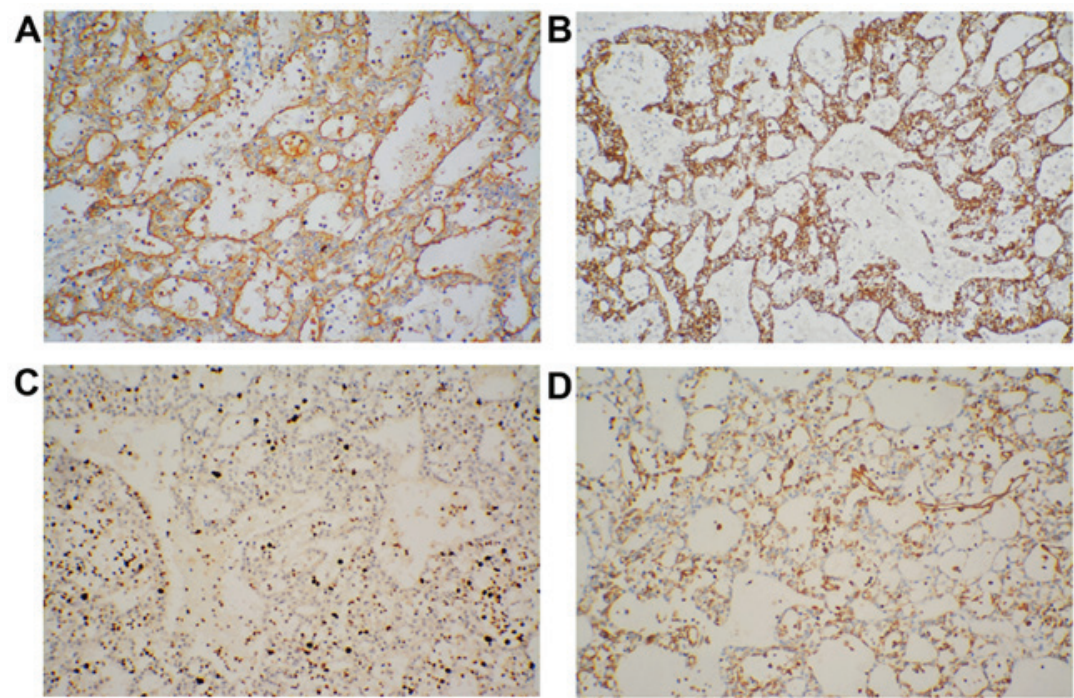

Figure 2. Immunohistochemical analysis of intraductal tubulopapillary neoplasm. (A) Partial positive results for cytokeratin, (B) mucin 1, (C) Ki-67 and (D) vimentin staining; magnification, x100.
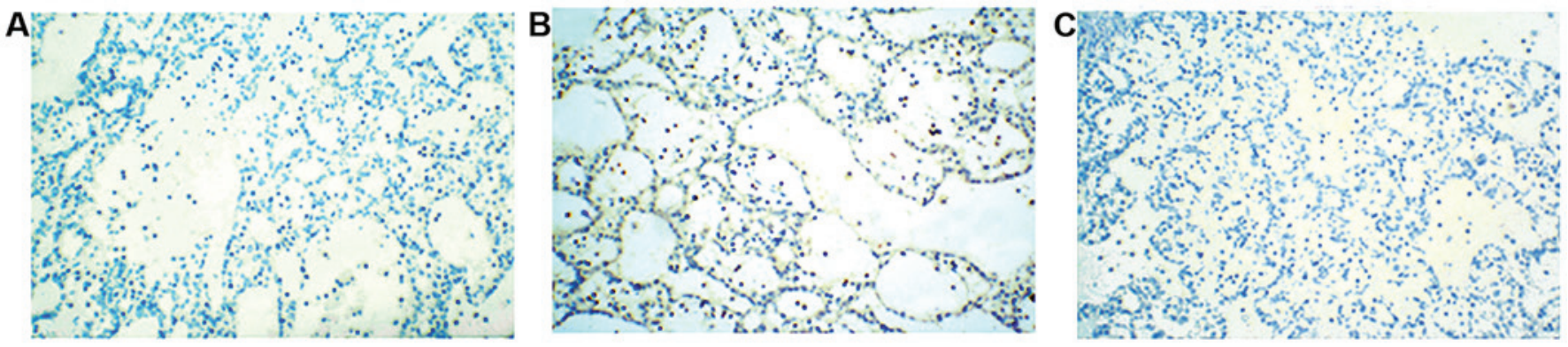

Figure 3. Immunohistochemical analysis of intraductal tubulopapillary pancreatic neoplasm showed negative results for (A) mucin 2, (B) CD56 and (C) chromogranin A staining; magnification, x100.

low incidence rate of ITPN, its diagnosis is often delayed and differential diagnosis may be challenging. The present study demonstrated a wide age range at onset for ITPN (35-82 years), with a median age of 55 years (2). There was no particular gender predilection (2). The majority of the patients exhibited mild clinical symptoms, such as abdominal pain, vomiting, weight loss and jaundice among others. In the laboratory examinations, the blood biochemical parameters and tumor markers had no clinical specificity. CT and MRI examinations revealed the pancreatic duct lesions. The most common site was the pancreatic head, accounting for $50 \%$, followed by the pancreatic body (34\%); the least common site was the pancreatic tail, accounting for only $15 \%$.

ITPN is an intraductal neoplasm that is accompanied by high-grade dysplasia. The gross pathological characteristics include uniform solid nodular polyps in the dilated pancreatic duct, with absence of mucin production (4). The size of the tumor is $\sim 0.5-15 \mathrm{~cm}$, and the pancreatic tissue surrounding the tumor is usually solid and hardened (4). Parts of the tumor exhibit tubular or back-to-back gland-like formations. In terms of morphology, the tumors display a sieve-like appearance rather than papillary formations in the pancreatic ducts. The tumor cells are cuboidal with a deeply stained nucleus, without mucus secretion in the cytoplasm. The proportion of patients with accompanying invasive carcinoma is $\sim 40 \%$.
Immunohistochemical analysis is helpful in diagnosing ITPNs. CK is an important marker differentiating the origin of the cell, as it is positive in tissues of epithelial origin. In the case reported herein, the immunohistochemical staining for CK was found to be positive, which confirmed that the neoplasm originated from the pancreatic duct epithelium (5). On this basis, other immunohistochemical targets have been used to distinguish the tumor origins, and included expression of MUC1 and MUC2, which are members of a family of high molecular weight proteins (5). Moreover, MUC1 encoded transmembrane mucin proteins and MUC2 encoded secretory mucin proteins. Previous case reports suggested that the tumors originated from pancreatic ductal epithelium when they stained positive for MUC1; however, MUC2-positive staining usually suggests that the tumor most likely arises from ampullary or colorectal tissues $(5,6)$. Therefore, in the case report presented herein, the CK-positive, MUC1-positive and MUC2-negative expression confirmed the ductal epithelial origin of the tumor.

The monoclonal antibody Ki-67 is an important marker in cell cycle analysis, reflecting the proliferative activity of the carcinoma (7). The expression of Ki-67 provides an important reference in distinguishing benign from malignant tumors. The Ki-67 index is associated with the histological grade of the tumor and patient prognosis, i.e., the higher the Ki-67 index, the higher the histological grade of the tumor and, thus, the poorer 
Table II. Clinicopathological characteristics of ITPN and IPMN.

Clinicopathological characteristics

Site

Clinical manifestations

Gross pathology

Histopathological characteristics

Immune phenotype

KRAS gene mutation

Prognosis
ITPN

IPMN

ITPN, intraductal tubulopapillary neoplasm; IPMN, intraductal papillary mucinous neoplasm; MUC, mucin; KRAS, Kirsten rat sarcoma viral oncogene homolog.

A

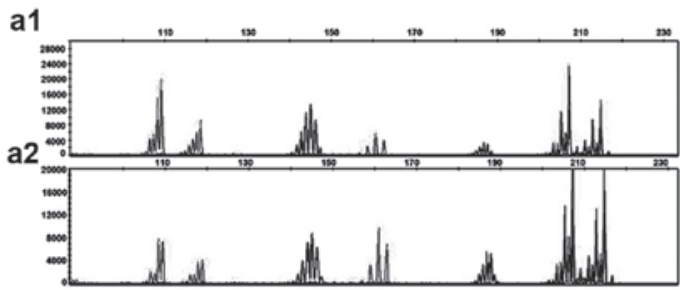

C

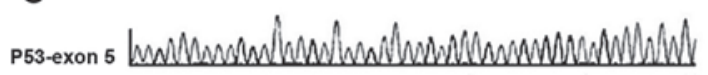

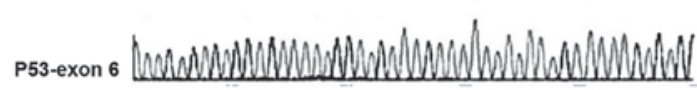

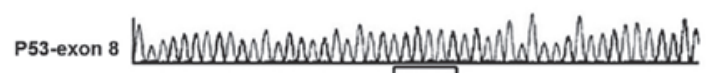

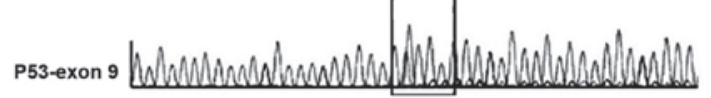

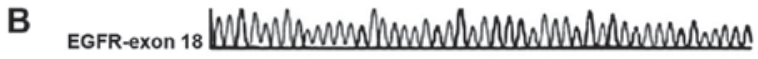

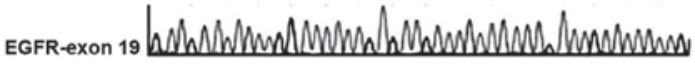

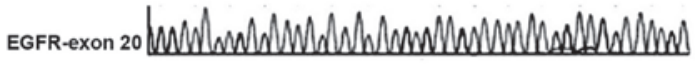

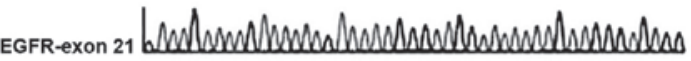

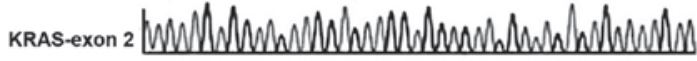

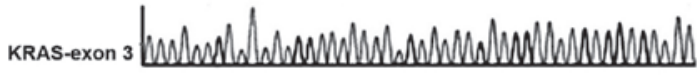

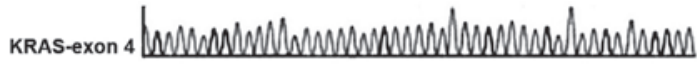

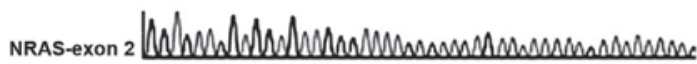

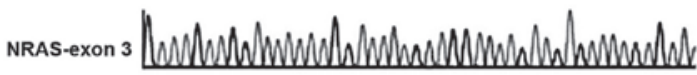

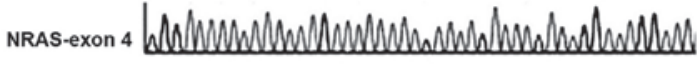

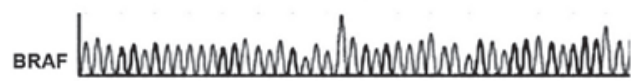

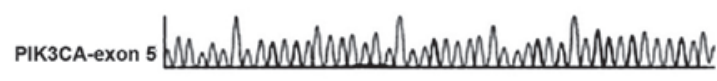

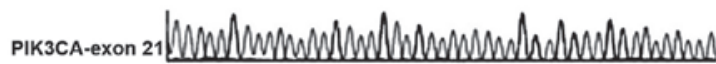

Figure 4. Gene mutation analysis for intraductal tubulopapillary neoplasm (ITPN) in this case. (A) Microsatellite instability analysis of ITPN results for (a1) normal and (a2) neoplastic tissues. (B) DNA sequence analysis of ITPN results for EGFR-KRAS/NRAS-BRAF pathway and PIK3CA pathway. (C) DNA sequence analysis of ITPN results for the P53 gene. EGFR, epidermal growth factor receptor; KRAS, Kirsten rat sarcoma viral oncogene homolog; NRAS, neuroblastoma RAS viral oncogene homolog; BRAF, B-Raf proto-oncogene; PI3KCA, phosphoinositide-3-kinase, catalytic subunit $\alpha$.

is the prognosis. In benign tumors of the pancreas, the $\mathrm{Ki}-67$ index is usually $<2 \%$. In our patient, the Ki-67 index was $20 \%$, which suggests a neoplastic tumor with low histological grade.

Subsequently, additional analyses were performed to exclude pancreatic endocrine tumors through the expression of CD56, CgA and vimentin. CD56 is a neural cell adhesion factor, which mainly characterizes tumors of neuroectodermal origin and is expressed by a number of endocrine tumors (8). $\mathrm{Cg}$ is divided into three subtypes, namely $\mathrm{A}, \mathrm{B}$ and $\mathrm{C}$, which comprise a group of soluble acidic proteins. $\mathrm{CgA}$ is most widely distributed in endocrine cells containing secretory granules and carcinomas of endocrine origin (9). Thus, $\mathrm{CgA}$ is currently used as an endocrine tumor marker. Vimentin is a marker of normal mesenchymal cells, which is also expressed in some epithelial cells or epithelial tumors (5). A number of pancreatic endocrine carcinomas are positive for the expression of $\mathrm{CgA}$ and CD56, but not necessarily vimentin. Therefore, the expression of vimentin, CD56 and CgA, suggests that the 
neoplastic origin in this case was not endocrine. CDX2 is a protein that is composed of 311 amino acids, containing a short sequence-specific binding region (10). Approximately $95 \%$ of colon or rectal carcinomas are CDX2-positive. However, MUC2 and CDX2 were not expressed in the intestinal epithelial specimens in the case reported herein.

According to previous studies, the EGFR pathway, including downstream proteins such as KRAS, NRAS and BRAF, is a common signal transduction pathway in digestive system tumors (11). EGFR is a type of glycoprotein, which belongs to the tyrosine kinase receptor family. Abnormal expression of EGFR is closely associated with proliferation, angiogenesis, invasion, metastasis and apoptosis of tumor cells (12). Under normal physiological conditions, the functional expression of the KRAS protein is rapidly inactivated following stimulation by EGFR. However, mutation of the KRAS gene leads to its sustained activation, as well as of the EGFR gene, which promotes tumor cell proliferation (13). Detection of KRAS gene mutations is clinically important as it plays an important role in identifying the specific characteristics of the tumor, and may help elucidate the process underlying the development of various cancers and administer the appropriate chemotherapy in clinical practice. In addition, RAF is activated by its upstream activator protein RAS, which binds to the Raf-1N-terminal. The RAS/RAF/MEK/ ERK pathway is one of the most important signal transduction pathways, as it is involved in several cellular physiological functions and also plays an important role in the pathogenesis and pathophysiology of a number of diseases (14). In this study, mutational analyses did not reveal any DNA sequence mutation in the common exons of EGFR, KRAS, NRAS or BRAF genes, indicating that the pathogenesis of ITPN is likely not related to the EGFR pathway.

P53 is a tumor suppressor gene, and P53 mutations occur in $~ 50 \%$ of all malignant tumors (15). The P53 protein is one of the transcription factors that regulates the cell cycle. Similar to all other tumor suppressor genes, the P53 gene plays a role in monitoring cell division under steady-state conditions (16). The P53 gene inhibits malignant transformation, determines the extent of variation of cellular DNA sequences and induces cellular repair by itself if the extent of cell damage is mild; by contrast, the P53 gene may induce apoptosis if the extent of cell damage is considerable (17).

Under conditions of mutation, the proper function of P53 is lost, as has been observed across a wide variety of mutations in a number of different tumors. The P53 gene is associated with $50 \%$ of human cancers, such as cancers of the liver, breast, bladder, gastric tissues, colon and prostate gland (18). The site of the P53 mutation in human cancer is usually found in the highly conserved regions that include positions 175, 248, 249, 282 and 273. However, the different types of P53 mutations found in tumors at different mutation sites, as is commonly found in colon and breast cancer, have a similar epidemiology. However, the P53 mutation spectrum is not consistent across tumor types. In the case reported herein, positive expression of P53 and DNA sequence analysis of the P53 gene introduced at a novel idea for the therapy of ITPN, which dominantly features P53 tumor gene therapy as a promising starting point that may offer new hope for combating ITPN.
Of note, IPMN may be confused with ITPN due to its similar morphology and clinical symptoms, such as jaundice and epigastric pain. However, the main characteristics of IPMN are mucin production that is accompanied by obvious ductal dilation, and the presence of mucous columnar epithelium, which are not present in ITPN. Moreover, serous cystadenoma (SCA), also referred to as microcystic adenoma, exhibited similarities to ITPN in terms of histological patterns. However, SCA often occurs in the pancreatic body or tail, the diameter of the tumor on cross-section is $1-2 \mathrm{~mm}$ and it is filled with a colorless fluid that differentiates it from ITPN.

\section{Acknowledgements}

The present study was supported by the Natural Foundation of Hubei Province (grant no. 2013CFB267) and the Wuhan Science and Technology Key Project (grant no. 2013060602010248).

\section{References}

1. Kasugai H, Tajiri T, Takehara Y, Mukai S, Tanaka J and Kudo SE: Intraductal tubulopapillary neoplasms of the pancreas: Case report and review of the literature. J Nippon Med Sch 80: 224-229, 2013

2. Yamaguchi H, Kuboki Y, Hatori T, Yamamoto M, Shimizu K, Shiratori K, Shibata N, Shimizu M and Furukawa T: The discrete nature and distinguishing molecular features of pancreatic intraductal tubulopapillary neoplasms and intraductal papillary mucinous neoplasms of the gastric type, pyloric gland variant. J Pathol 231: 335-341, 2013.

3. Kölby D, Thilén J, Andersson R, Sasor A and Ansari D: Multifocal intraductal tubulopapillary neoplasm of the pancreas with total pancreatectomy: Report of a case and review of literature. Int $\mathbf{J}$ Clin Exp Pathol 8: 9672-9680, 2015.

4. Yamaguchi H, Shimizu M, Ban S, Koyama I, Hatori T, Fujita I, Yamamoto M, Kawamura S, Kobayashi M, Ishida K, et al: Intraductal tubulopapillary neoplasms of the pancreas distinct from pancreatic intraepithelial neoplasia and intraductal papillary mucinous neoplasms. Am J Surg Pathol 33: 1164-1172, 2009.

5. Modi Y, Shaaban H, Gauchan D, Maroules M, Parikh N and Guron G: Primary clear cell ductal adenocarcinoma of the pancreas: A case report and clinicopathologic literature review. J Cancer Res Ther 10: 773-776, 2014.

6. Yokoyama S, Kitamoto S, Higashi M, Goto Y, Hara T, Ikebe D, Yamaguchi T, Arisaka Y, Niihara T, Nishimata H, et al: Diagnosis of pancreatic neoplasms using a novel method of DNA methylation analysis of mucin expression in pancreatic juice. PLoS One 9: e93760, 2014.

7. Garcia-Carracedo D, Yu CC, Akhavan N, Fine SA, Schönleben F, Maehara N, Karg DC, Xie C, Qiu W, Fine RL, et al: Smad4 loss synergizes with TGF $\alpha$ overexpression in promoting pancreatic metaplasia, PanIN development and fibrosis. PLoS One 10: e0120851, 2015.

8. Stelow EB, Shaco-Levy R, Bao F, Garcia J and Klimstra DS: Pancreatic acinar cell carcinomas with prominent ductal differentiation: Mixed acinar ductal carcinoma and mixed acinar endocrine ductal carcinoma. Am J Surg Pathol 34: 510-518, 2010.

9. Gurung B, Hua X, Runske M, Bennett B, LiVolsi V, Roses R, Fraker DA and Metz DC: PTCH 1 staining of pancreatic neuroendocrine tumor (PNET) samples from patients with and without multiple endocrine neoplasia (MEN-1) syndrome reveals a potential therapeutic target. Cancer Biol Ther 16: 219-224, 2015.

10. Kumari N, Prabha K, Singh RK, Baitha DK and Krishnani N: Intestinal and pancreatobiliary differentiation in periampullary carcinoma: The role of immunohistochemistry. Hum Pathol 44: 2213-2219, 2013.

11. Nagathihalli NS, Beesetty Y, Lee W, Washington MK, Chen X, Lockhart AC and Merchant NB: Novel mechanistic insights into ectodomain shedding of EGFR ligands amphiregulin and TGF- $\alpha$ : Impact on gastrointestinal cancers driven by secondary bile acids. Cancer Res 74: 2062-2072, 2014. 
12. Sreekumar BK, Belinsky GS, Einwachter H, Rhim AD, Schmid R and Chung C: Polarization of the vacuolar adenosine triphosphatase delineates a transition to high-grade pancreatic intraepithelial neoplasm lesions. Pancreas 43: 1256-1263, 2014.

13. Mackinnon AC Jr, Luevano A, de Araujo LC, Rao N, Le M and Suster S: Cribriform adenocarcinoma of the lung: Clinicopathologic, immunohistochemical, and molecular analysis of 15 cases of a distinctive morphologic subtype of lung adenocarcinoma. Mod Pathol 27: 1063-1072, 2014.

14. Schultz NA, Roslind A, Christensen IJ, Horn T, Høgdall E, Pedersen LN, Kruhøffer M, Burcharth F, Wøjdemann M and Johansen JS: Frequencies and prognostic role of KRAS and BRAF mutations in patients with localized pancreatic and ampullary adenocarcinomas. Pancreas 41: 759-766, 2012.
15. Guan H, Gurda G, Lennon AM, Hruban RH and Erozan YS: Intraductal tubulopapillary neoplasm of the pancreas on fine needle aspiration: Case report with differential diagnosis. Diagn Cytopathol 42: 156-160, 2014.

16. Navas C, Hernández-Porras I, Schuhmacher AJ, Sibilia M, Guerra $\mathrm{C}$ and Barbacid M: EGF receptor signaling is essential for k-ras oncogene-driven pancreatic ductal adenocarcinoma. Cancer Cell 22: 318-330, 2012.

17. Shin SH, Kim SC, Hong SM, Kim YH, Song KB, Park KM and Lee YJ: Genetic alterations of K-ras, p53, c-erbB-2, and DPC4 in pancreatic ductal adenocarcinoma and their correlation with patient survival. Pancreas 42: 216-222, 2013.

18. Søreide K and Sund M: Epidemiological-molecular evidence of metabolic reprogramming on proliferation, autophagy and cell signaling in pancreas cancer. Cancer Lett 356: 281-288, 2015. 